\title{
Systemuntersuchungen - Fahrleitung, Schleifleiste, Stromabnehmer
}

\section{Zick-Zack-Lage}

\author{
Mitteilung der Fa. DTK Deutzer Technische Kohle GmbH im TGZ Wildau
}

Dipl.-Chem. Manfred Deutzer, Dipl.-Ing. Torsten Richter

\section{Einleitung}

Die störungsfreie Stromentnahme aus Fahrleitungen wird von zahlreichen Einflußgrößen bestimmt. Bei den meisten elektrisch betriebenen Fahrzeugen erfolgt die Stromentnahme mit Kohleschleifleisten. Die Schleifleisten werden von unten gegen die Fahrleitung gedrïckt und gleiten an der Fahrleitung entlang. Im Betrieb muß die Schleifleiste bei allen Fahrgeschwindigkeiten und unter allen Witterungsbedingungen den Strom sicher aus der Fahrleitung entnehmen.

Bei einer zu hohen Anpreßkraft wird die Fahrleitung angehoben. Dabei können Klemmen und andere Bauteile der Fahrleitung abkippen. Das fuihrt zu Schlägen zwischen Fahrleitung und Schleifleiste, die Störungen im Fahrbetrieb verursachen. Gleichzeitig wird der mechanische Verschleiß der Kohlekörper bedeutend erhöht. Bei einer zu geringen Anpreßkraft wird der Stromiibergang gestört. Es entstehen zahlreiche kurzzeitige Kontaktunterbrechungen bzw. Kontaktverschlechterungen. Die hohe elektrische Leistung wird in diesem Fall über partielle Lichtbögen oder über die noch vorhandenen Engebereiche von der Fahrleitung/Stromschiene zur Schleifkohle übertragen. Die Lichtbögen werden als Bügelfeuer bezeichnet. Die Kupferfahrleitung erreicht dabei an bestimmten Punkten der Kontaktfläche eine Temperatur von mehreren hundert Grad. Bereits bei $190{ }^{\circ} \mathrm{C}$ erfolgt die Entfestigung durch den Rekristallisationsprozeß. Es entstehen auf der Kontaktfläche der Fahrleitung feste und weiche Bereiche. Die Folge ist ein ungleichmäßiger Fahrleitungsverschleiß. Auch die Schleifkohle wird durch die Lichtbögen überhitzt. Die Schleifkohle verschleißt zusätzlich durch Oxydation.

Eine Überhitzung der Schleifkohle kann aber auch durch die Stromentnahme an ein und der selben Stelle der Kohle erfolgen. Bereits bei einer geringen elektrischen Belastung wird die Kontaktstelle erheblich aufgeheizt. Es können Temperaturen von weit über $600{ }^{\circ} \mathrm{C}$ erreicht werden. An den iiberhitzten Stellen im Kohlekörper entstehen später Querrillen.

Während des Betriebes werden die Fahrzeuge ständig beschleunigt und abgebremst. Auf die Wippe/Schleifleisten wirkt hierdurch ein Trägheitsmoment, so daß mal die eine und mal die andere Schleifleiste einen ungenügenden elektrischen Kontakt mit der Fahrleitung besitzt. Die Schleifleisten verschleißen ungleichmäßig. An bestimmten Stellen muß die Fahrleitungshöhe verringert werden um z. B. Brïckendurchfahrten zu er- möglichen. Die Schleifleisten muissen auch bei sehr unterschiedlichen Arbeitshöhen immer ausreichend gut mit der Fahrleitung kontaktieren.

Um die Schäden am System Fahrleitung/Stromabnehmer/Schleifkontakt möglichst gering zu halten, werden regelmäßig Inspektionen der Fahrleitung und der Schleifleisten durchgefuihrt. Zusätzlich werden zu diesem Zweck in den Stromabnehmer Sollbruchstellen eingebaut.

Von der Firma Deutzer-Technische Kohle (DTK) wurden die Fahrleitung, der Stromabnehmer und die Schleifleisten untersucht und Methoden entwickelt, mit denen im normalen Fahrbetrieb Fehler so früh erkannt werden können, daß rechtzeitig, vor einem großen Schaden, geeignete Maßnahmen eingeleitet werden können. Andere DTK-Systeme dienen zum Beheben von Störungen „DTK-Glattschleifsystem für Stromschienen und Fahrleitungen" oder zur optimalen Einstellung der Kontaktpreßkraft - „DTK-System-Fahrdynamik“.

\section{Herstellung und Reaktivität der Kohleschleifkörper}

Zur Einschätzung des Systems Fahrleitung/Stromabnehmer/Schleifkontakt ist die Kenntnis vom Verhalten der Kontaktpartner erforderlich. Der Kontaktpartner „Kohleschleifleiste“ spielt hierbei für das Gesamtsystem eine entscheidende Rolle. Schließlich soll der Kohlekörper unter allen Witterungsbedingungen und bei allen Fahrgeschwindigkeiten einen guten elektrischen Kontakt zur Fahrleitung ermöglichen. Der Abrieb von der Fahrleitung soll dabei möglichst gering sein.

Bei der Herstellung der Kohleschleifkörper werden Kokskörner unterschiedlicher Größe mit dem Bindemittel (Pech) bei einer Temperatur von $100^{\circ} \mathrm{C}$ bis $160^{\circ} \mathrm{C}$ gemischt. Hierbei bildet sich um die Kokskörner ein Bindemittelfilm. Das warme plastische Koks-Bindemittel-Gemisch wird dann als Strang gepreßt und anschließend gebrannt. Beim Brennen wird der Kohlekörper langsam aufgeheizt. Hierbei entweichen aus dem Bindemittel im Inneren des Strangs gasförmige Bestandteile. Diese müssen ohne den Strang aufzureißen an die geometrische Oberfläche gelangen. Hierdurch ist der gesamte Kohlekörper porös. Die Porosität liegt bei ca. 10 bis $20 \%$. Der während des Einsatzes der Schleifkohle zur Oxireaktion erforderliche Luftsauerstoff kann relativ einfach in den Kohlekörper eindringen. Um die Porosität und den Reib- 
wert zu verringern werden die Schleifkohlen häufig imprägniert oder zusätzlich eingewachst. Der Kohlenstoffanteil im Bindemittel bildet Brücken zwischen den Kokskörnern. Die Bindemittel-Kohlenstoffbrücken sind sehr viel reaktiver gegenuiber Luftsauerstoff als die Kokskörner und werden bei einer thermischen Überlastung zuerst geschwächt.

Die Kinetik der Reaktivität von Kohleschleifleisten während der Stromabnahme im Fahrbetrieb wird durch eine Reihe von Faktoren bestimmt. Diese sind unter anderem die Dichte/Porosität und Störungen in der Struktur des Kohlekörpers, der Diffusionsprozeß, Verunreinigungen im Kohlegefüge, Rohstoffart und Zusammensetzung, sowie die Technologie bei der Herstellung der Schleifkohlekörper wie z.B. die Brennendtemperatur.

Die Reaktionsgeschwindigkeit von Schleifkohlen wird in unterschiedliche Temperaturbereiche unterteilt. Im unteren Temperaturbereich bis ca. $450{ }^{\circ} \mathrm{C}$ ist die Reaktion ein den Kohlekörper durchdringender Prozeß. Der Transport der Gasmolekiile aus dem Volumen des Kohlekörpers zur geometrischen Oberfläche und der Transport von Sauerstoffmolekiilen zu den reaktiven Plätzen in der Kohle bestimmt die Reaktionsgeschwindigkeit. Der Gastransport erfolgt bei einer konstanten Temperatur durch molekulare Diffusion durch die Grenzschicht und durch die Poren (Defektstrukturen) des Kohlekörpers. Die Gasdiffusion ist größer als die Reaktionsgeschwindigkeit. Bei höheren Temperaturen gelangt der reaktive Sauerstoff nicht mehr in den Kohlekörper. Er wird sofort an der Oberfläche der Schleifkohle $\mathrm{zu} \mathrm{CO} / \mathrm{CO}_{2}$ umgesetzt.

Im Betrieb wird die Schleifleiste an der Kontaktstelle Fahrleitung-Kohlekörper und im Kohlekörper in Bereichen sehr hoher Stromdichte erwärmt. Durch diese Erwärmung findet die o. g. Niedertemperaturoxidation des Kohlekörpers statt. Die Brücken zwischen den Kokskörnern (Sekundärkoksgeruist) werden geschwächt. Im Fahrbetrieb wird an diesen Stellen sehr viel mehr Material aus der Kohleleiste herausgeschliffen als an den Stellen, die nicht uiberlastet wurden. Die Folge sind Querrillen im Schleifkohlekörper. Gleichzeitig wird der Kohlekörper durch den Fahrtwind gekiihlt. Dabei werden neue reaktive Moleküle in den Kohlekörper gesogen. Bei der nächsten thermischen Belastung wird der mit Luftsauerstoff zu $\mathrm{CO} / \mathrm{CO}_{2}$ umgesetzte Kohlenstoff durch die Ausdehnung des Gases aus dem Porenvolumen gepreßt. Somit entsteht ein zusätzlicher Pumpvorgang, der dafür sorgt, daß immer ausreichend Sauerstoff an den reaktiven Plätzen im Kohlekörper vorhanden ist.

\section{Systemuntersuchung Fahrleitung - Schleifleiste}

\subsection{Elektrische Untersuchungen}

Von DTK wurde über die Kontaktflächenerwärmung während der Stromentnahme aus der Fahrleitung berichtet $^{1}$. Dazu wurde mittels Wärmebildkamera der Kontakt zwischen der Fahrleitung und der Schleifleiste bei der Entnahme von nur $4 \mathrm{~kW}$ untersucht. Bereits bei der Übertragung kleinster elektrischer Leistungen wird die Kontaktstelle zwischen der Fahrleitung und der
Schleifkohle um mehr als 50 Grad erwärmt. Moderne Fahrzeuge benötigen jedoch eine 100 fach größere elektrische Leistung.

\subsection{Mechanischer Verschleiß}

Der mechanische Verschleiß der Fahrleitung und der Schleifleisten wird durch die Reibung bei der Kontaktierung und durch Stöße zwischen den Kontaktpartnern verursacht. Der minimale Abrieb der Schleifkohle bei durchschnittlicher Schleifkohlehärte liegt bei ca. 0,5 bis $2 \mathrm{~mm}^{3} / \mathrm{km}$. Dieser Wert wird in Abhängigkeit von den physikalischen Werten des Schleifkohlekörpers, von der elektrischen Belastung der Schleifleiste im Fahrbetrieb, von den Witterungs- und Umweltbedingungen usw. mehr oder weniger stark beeinflußt. Bei sehr harten Schleifkohlen wird der Verschleiß auf die Seite der Fahrleitung verschoben. Die Schleifkohlen erreichen zwar eine höhere Laufleistung, die Lebensdauer der Fahrleitung nimmt jedoch stark ab.

Bei starken Stößen zwischen dem Schleifkohlekörper und der Fahrleitung kann die Schleifkohle sofort ausfallen. Die Stöße und Schläge zwischen der Fahrleitung und der Schleifkohle fuihren zu Strukturschäden innerhalb des Schleifkohlekörpers. Straßenbahnen befahren an einem Tag ein und die selbe Linie zahlreiche Male. Dabei wird bei einer Störung an der Fahrleitung ein Schlag auf die Schleifkohle ausgeübt, der sich bei jeder Durchfahrt der Bahn an diesem Streckenpunkt wiederholt. Bei den ersten Schlägen werden im Kohlekörper meist nur geringe Strukturschäden verursacht. Die weiteren Schläge fuihren dann zu großflächigen Ausplatzungen, zu Kohlebrüchen und auch zur Bildung von Querrillen.

Beim Beschleunigen oder beim Abbremsen werden im System Stromabnehmer/ Schleifleisten durch die Trägheit Schwingungen erzeugt, die zu einer ständig wechselnden Kontaktqualität führen. Dies kann die Ursache für die Entstehung von Rattermarken in der Fahrleitung und zur Bildung von Querrillen in der Schleifkohle sein.

\subsubsection{Stoßuntersuchung}

Bei zahlreichen Verkehrsunternehmen und Vollbahnbetreibern in West- und Osteuropa wurde das DTK-System „Stoß“ zur Fehlersuche eingesetzt. Es wurden dabei turnusmäßige Untersuchungen und Abnahmeprïfungen von neu installierten Fahrleitungen durchgefuihrt.

Bei einigen Prüfungen mit dem DTK-System-Stoß wurden von den Verkehrsbetrieben Vergleichsuntersuchungen mit eigenen Mitteln durchgefuihrt. Dabei wurden nur ca. 2 bis $5 \%$ der vom DTK-System gefundenen Fehler ermittelt. Eine anschließend durchgefuihrte Sichtkontrolle von der Bühne eines Turmwagens bestätigte jedoch alle mit dem DTK-System gefundenen Fehlerstellen. Die zahlreichen Untersuchungen haben bewiesen, daß Stöße zwischen Schleifleiste und Fahrleitung nur in den seltensten Fällen durch Sichtprüfung erkannt werden können.

Bei der DB AG wird die Fahrleitungsseitenlage und auftretende Schläge zwischen der Fahrleitung und der Schleifleiste für die durchgehenden Hauptgleise alle 12 
Monate geprüft, dabei wird mit einem Oberleitungs-Regulierungswagen bei einer Geschwindigkeit von $40 \mathrm{~km} / \mathrm{h}$ eine Strecke abgefahren. Die statische Anpreßkraft der Schleifleisten gegen die Fahrleitung beträgt 150 N. In der Beobachtungskanzel des Regulierungsfahrzeuges sitzen zwei erfahrene und zuverlässige Mitarbeiter, die den Lauf des Fahrdrahtes und das Zusammenwirken mit dem Stromabnehmer beobachten. „Sollte das Ergebnis „kein Fehler" lauten, sagt das nur, daß kein Fehler gefunden wurde, nicht jedoch, daß die Anlage fehlerfrei ist"

Das DTK-System erfaßt unabhängig von der Qualität des Prüfpersonals und anderen äußeren Bedingungen alle auftretenden Schläge und Stöße. Bei einem Ergebnis „kein Fehler" ist die Fahrleitung störstellenfrei.

Das DTK-Stoßmeßsystem besteht aus umweltfreundlichen DTK-Schleifleisten mit eingebauten Sensoren. Die Signale der Sensoren gelangen uiber Lichtwellenleiter in das Fahrzeuginnere und werden dort von einem Computer und einem Videogerät aufgezeichnet. Während oder nach der Prüfung können die Stellen ermittelt werden, an denen zwischen der Fahrleitung und der Schleifleiste ein Stoß aufgetreten ist. Die Stöße werden in „sofort zu beheben“, ,kurzfristig zu beheben“, „längerfristig zu beheben" unterteilt. Somit wird der Fahrleitungsinstandhaltung die Möglichkeit gegeben, vorrangig die wichtigsten Problemstellen zu beseitigen. Über den Aufbau und das Funktionsprinzip des DTKMeßsystems wurde in verschiedenen Veröffentlichungen bereits berichtet ${ }^{3,4}$. Aus diesem Grund soll an dieser Stelle nur auf einige Ergebnisse von Untersuchungen eingegangen werden.

Bei der Abnahmeprüfung eines neu erstellten Fahrleitungsabschnittes von ca. 2,5 km wurde bei einem Verkehrsbetrieb eine Störstelle gefunden, die kurzfristig behoben werden mußte. Bei einem anderen Verkehrsbetrieb wurde eine neu aufgebaute moderne Fahrleitung von $17 \mathrm{~km}$ untersucht. Dabei wurden 8 Störstellen der Kategorie „sofort zu beheben“ gefunden. Weitere 15 Störstellen mußten kurzfristig instandgesetzt werden. Mit Hilfe der DTK-Stoßuntersuchung wurden die Störstellen gefunden und von der Errichterfirma gleich nach der Prüfung beseitigt.

Bei einem anderen Verkehrsbetrieb wurde eine Fahrleitungsstrecke (Hochkette) von ca. $40 \mathrm{~km}$ untersucht. Hierbei wurden 37 sofort zu behebende Störstellen ermittelt. 15 Störstellen mußten kurzfristig reguliert werden.

Die Fahrleitungen wurden bei über 60 Verkehrsunternehmen in 13 europäischen Ländern gepriift. Bei zahlreichen Unternehmen wurde die Abnahmeprüfung für neu erstellte Fahrleitungen durchgefuihrt. Keine einzige Prüfung konnte mit dem Prüfergebnis „kein Fehler“ abgeschlossen werden.

Mit dem DTK-System-Stoß besteht aber auch die Möglichkeit optimale Einbaubedingungen für Streckentrenner bzw. andere Bauteile zu ermitteln.

\subsubsection{Zick-Zack-Lage}

Eine ausreichende Zick-Zack-Lage der Fahrleitung ist für einen störungsfreien Fahrbetrieb, für eine optimale Ausnutzung der Schleifkohlen und für einen geringen Verschleiß der Fahrleitung unerläßlich. Bei einem zu geringen Zick-Zack erfolgt die Stromentnahme an ein und der selben Stelle am Kohlekörper. Dieser wird dadurch elektrisch (thermisch) und mechanisch überlastet. Die Folge sind Querrillen in der Schleifkohle. Die Schleifleisten miissen dann vorzeitig gewechselt werden um Gewaltschäden zu vermeiden. Der Mehraufwand für zusätzliche Schleifleisten und der Arbeitsaufwand für die Wartung und Instandhaltung ist beträchtlich.

Die Ermittlung der Fahrleitungslage ausgehend vom Gleis mit einem Meßkreuz oder mit Meßverfahren die beriihrungslos arbeiten bestimmt nur die Lage über der Fahrschiene, sie berïcksichtigt nicht die Eigenschaften des Fahrzeuges, des Stromabnehmers und des Gleises.

Ein DTK-System zur Zick-Zack-Lage Ermittlung5 basiert auf der Anwendung von Lichtwellenleitern als optische Schalter. Dabei werden an der Schleifleiste zahlreiche Lichtwellenleiterenden befestigt, deren Ausgang nach oben gerichtet ist. Im Fahrbetrieb wird dann der jeweilige Lichtwellenleiterausgang durch die Fahrleitung abgedeckt. Das Tageslicht kann nicht in den Lichtwellenleiter eingekoppelt werden. Das erhaltene Signal wird im Fahrzeug mittels PC ausgewertet und somit die momentane Lage der Fahrleitung angezeigt. Hierdurch kann jedoch die Fahrleitungslage nur an diskreten Punkten ermittelt werden.

Diese Methode ist auch sehr aufwendig durch die Verwendung von vielen Lichtwellenleitern mit je ca. $6 \mathrm{~m}$ bis $7 \mathrm{~m}$ Länge und für jeden Meßpunkt eine gesonderte Schaltelektronik. Bei Prüfungen im Tunnel muß zusätzlich eine Lichtquelle installiert werden. Bei ungünstigen Lichtverhältnissen ist eine Messung nahezu unmöglich. Das Rechnerprogramm ist so aufgebaut, daß in Lichtwellenleiter einfallende Schatten durch Bäume, Brüicken, Masten oder Abspannungen erkannt und elimeniert werden. Ein besonderes Problem stellt die Verschmutzung der Lichtwellenleiter-Eingänge dar.

Häufig wird die Fahrleitungsseitenlage mit einem einfachen Meßlineal am Stromabnehmer oder mit speziellen Meßschleifleisten mit Näherungssensoren ermittelt, die stets auf besonderen Prüffahrzeugen installiert sind. Diese Schleifleisten sind durch den Anbau des Meßlineals oder der zahlreichen Nährungssensoren uiber die gesamte Länge der Leiste sehr schwer. Da die Signale bei der Verwendung von Näherungssensoren über elektrische Leitungen ins Fahrzeuginnere gefuihrt werden, muß gesichert sein, daß das Fahrleitungspotential nicht in das Fahrzeug gelangen kann. Der Aufwand hierfuir ist sehr groß. Eine kontinuierliche Lageerfassung ist oft nicht möglich oder vorgesehen.

Die Lage kann mit diesem System auf ca. $50 \mathrm{~mm}$ genau ermittelt werden. Die Meßergebnisse beziehen sich aber nur auf das Meßfahrzeug. Bei normalen Linienfahrzeugen kann die Lage der Fahrleitung auf der Schleifleiste von den Meßdaten erheblich abweichen. Hinzu 
kommt, daß die Fahrzeugflotte der meisten Verkehrsunternehmen aus sehr unterschiedlichen Fahrzeugen besteht, die sich z.B. in der seitlichen Stabilität stark untereinander unterscheiden.

Von DTK wurde der Wunsch der Verkehrsunternehmen nach einem einfachen, kostenguinstigen und schnell zu installierenden System zur Messung der Fahrleitungslage auf der Schleifleiste beriicksichtigt und ein neues System entwickelt ${ }^{6}$, das

- auf jedes beliebige Fahrzeug in kuirzester Zeit aufgebaut werden kann

- die Lage der Fahrleitung auf der Schleifleiste im normalen Fahrbetrieb ermittelt

- eine sehr genaue Bestimmung der Fahrdrahtlage ermöglicht

- zur regelmäßigen Pruifung und zu Abnahmepruifungen von neu installierten Fahrleitungen geeignet ist

- die Masse der Schleifleiste nur gering beeinflußt

- keine besonderen Sicherheitsmaßnahmen erfordert

- für Dienstleistungen durch DTK und somit auch für kleine Verkehrsunternehmen geeignet ist

- alle Fragestellungen zur Fahrleitungsseitenlage und Höhenlage beantwortet.

Fragestellungen zur Fahrleitung können z. B. sein:

- Wo ist der Zick-Zack kleiner als ein vorgegebener Wert?

- Wo werden Grenzlagen ïberschritten ?

- Auf welchen Schleifkohlebereichen erfolgt die Kontaktierung wie lange und wie häufig ?

- Wie ist die Lageverteilung der Fahrleitung fuir beliebige Streckenabschnitte ?

Wie groß ist der Zick-Zack an beliebigen Stellen ?

- Wie groß ist die Lageänderung auf beliebigen Strekkenabschitten?

- Wie hat sich die Lage in einem Zeitabschnitt (Sommer/Winter) verändert ?

Das System kann auch im Zusammenhang mit anderen DTK-Systemen z.B. mit der Stoßprifung der Fahrleitung oder mit dem System zur Ermittlung des statischen und fahrdynamischen Verhaltens des Stromabnehmers eingesetzt werden. Besonders aussagekräftige Ergebnisse werden durch die Kombination der DTK-Systeme „ZickZack-Lage“ und „elektrische Belastung der Kontaktstelle" erhalten. Hierbei wird beriicksichtigt, daß im Anfahrbereich bzw. bei Fahrzeugen mit Ruickspeisung beim Bremsen sehr hohe elektrische Leistungen ibertragen werden müssen. Der Zick-Zack der Fahrleitung ist an derartigen Streckenabschnitten oft nicht größer als bei Fahrleitungsabschnitten an denen geringe elektrische Leistungen übertragen werden müssen.

\section{Aufbau des Meßsystems}

Entsprechend der Bauform und Ausfiihrung der vorhandenen Wippe und des Pantographen können die DTK-Meßschleifleisten angepaßt werden. Im Bild 1 ist eine Wippe mit DTK-Schleifleisten und DTK-Meßsystem dargestellt.
In dieser Ausführung besitzt die Schleifleiste zur Erfassung der Lage eine Kohlebreite von $42 \mathrm{~mm}$ und die zweite Schleifleiste auf der Wippe eine Kohlebreite von 62 mm. Da an der schmalen Leiste zusätzlich die Meßwertaufnahme angebracht ist, haben beide Leisten die gleiche Masse. Das Wippensystem befindet sich somit wieder im Gleichgewicht.

Die DTK-Meßschleifleiste besteht aus einer DTK-Schleifleiste mit den Endstuicken. Seitlich an der Schleifleiste sind zwei Meßlineale angebracht. An

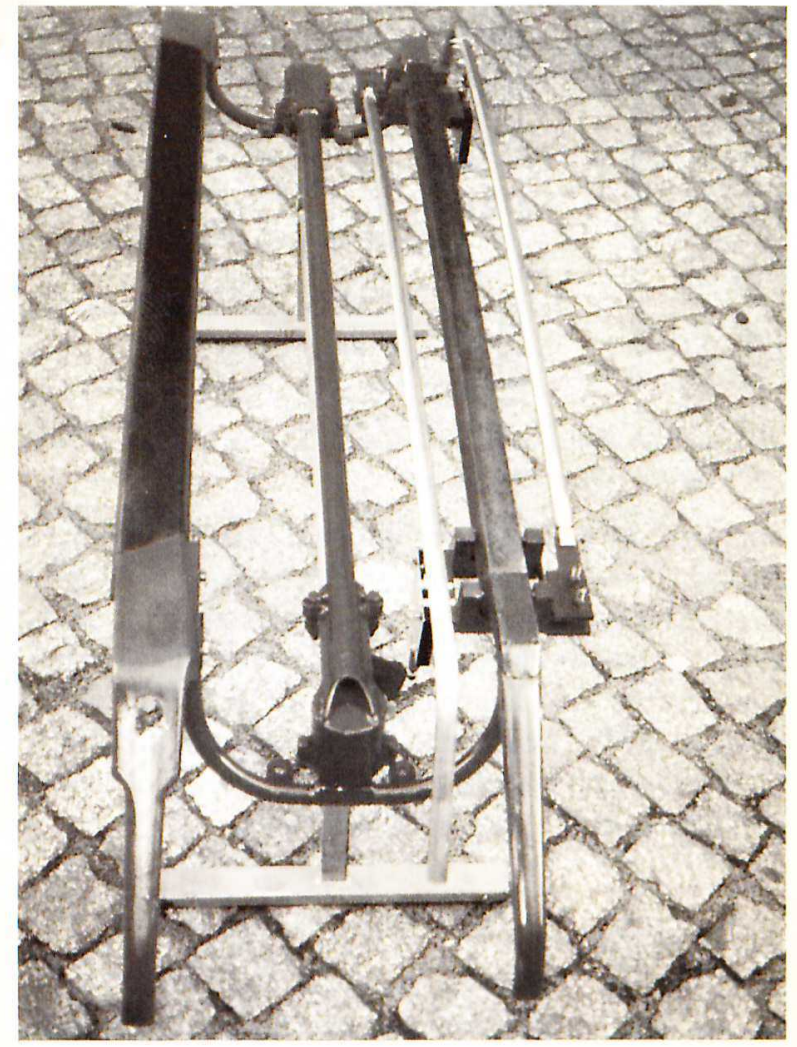

Bild 1 DTK-Wippe mit Meßleiste für die Zick-Zack-Lage

den Meßlinealen sind die optischen Meßaufnehmer befestigt. Sie drücken die Meßlineale gegen die Fahrleitung. Die Meßlineale ragen geringfügig über das $\mathrm{Ni}$ veau der Schleifkohlefläche der Schleifleiste. Sie sind an den Enden so gebogen, daß im Einsatz die Fahrleitung problemlos auf die Lineale gleiten kann. Während der Prïfung werden die Lineale durch die Fahrleitung auf das Niveau der Kohleschleifflächen gedrückt. Dabei wird der jeweilige Meßaufnehmer auf den für die Lage der Fahrleitung bestimmten Wert eingestellt. Die ermittelten Lagewerte werden iiber Lichtwellenleiter in das Fahrzeuginnere zur Auswerteinrichtung übertragen. Durch die Verwendung von Lichtwellenleitern ist eine galvanische Entkopplung zwischen Fahrleitungspotential und Fahrzeug/Erdpotential gewährleistet. An den Meßlinealen können Leitbleche angebracht sein, die an Kreuzungen das Lineal nach unten drücken. 


\section{Software}

Für das DTK-System „Zick-Zack-Lage“ wurde eine spezielle Software entwickelt. Vor Beginn der Entwicklung wurden zukuinftige Benutzer nach Ihren Wünschen für das Handling des Systems befragt. Alle Wünsche der Anwender wurden berïcksichtigt. Besondere Wünsche und Forderungen von Anwendern können auch noch nachträglich in die Software eingearbeitet werden. Die Software wird bei Bedarf auch in andere vorhandene Meßsysteme integriert, z. B. kann sie an Schleifleisten mit Näherungssensoren angepaßt werden.

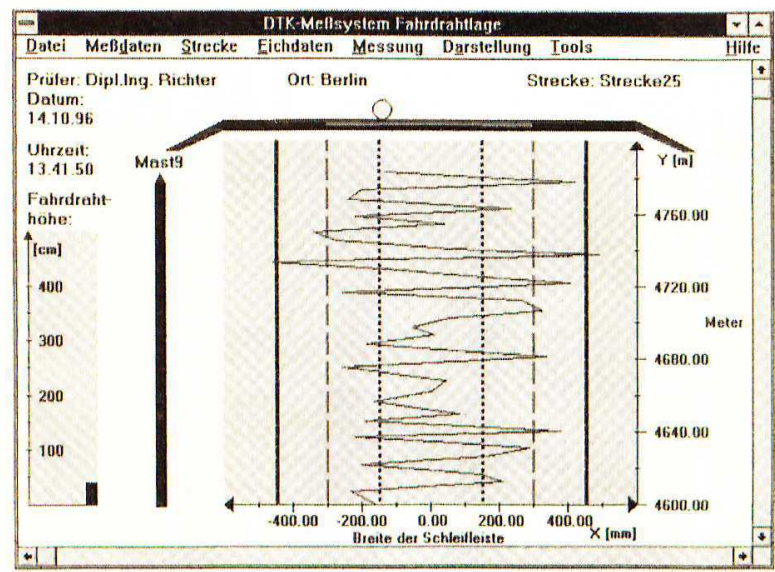

Bild 2 Monitor während der Prüfung

Während der Prüfung wird auf dem Monitor - Bild 2 die Lage der Fahrleitung, die Überschreitung von Grenzwerten der Fahrdrahtseitenlage und der zugehörige Ort sowie markante Punkte der Prüfstrecke wie Kreuzungen, Masten usw. angezeigt. Computerinterne Daten wie z.B. Schnittstelle oder Format der Daten zur Weiterverarbeitung in unterschiedlichen Datenbanken können nach Wunsch eingestellt werden ${ }^{7}$.

\section{Auswertung}

Da die Datenerfassung in unterschiedlichen Formen erfolgen kann, besteht die Möglichkeit der Auswertung mit verschiedenen Datenbanken oder Tabellenkalkulationsprogrammen z.B. Exel, d-Base. Es kann beispielsweise die Ist-Fahrleitungslage mit einer vorgegebenen Soll-Lage verglichen werden. Aus der Differenz werden eventuell erforderliche Regulierungsmaßnahmen abge-

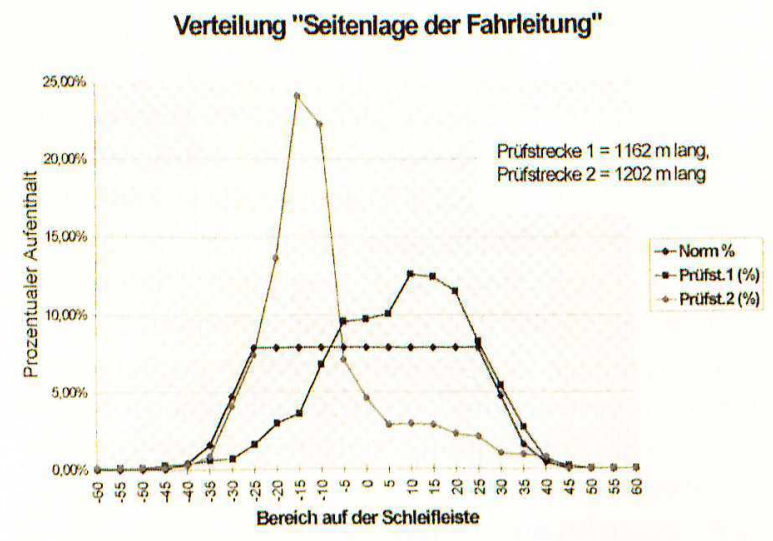

Bild 3 Vergleich zwischen Soll- und Ist-Fahrleitungslage leitet. Mit der Lageveränderung kann dann die Laufleistung der Schleifleisten beträchtlich erhöht werden. Das Bild 3 stellt die Soll-Lage und die Ist-Lage für unterschiedliche Streckenabschnitte dar.

Zur Auswertung wurden die Daten in die Tabellenkalkulation „Exel“ eingelesen. Die frei gewählte Prïstrecke 1 ist $1162 \mathrm{~m}$ lang und die Prüfstrecke 2 ist $1202 \mathrm{~m}$ lang. Bei einem Zick-Zack entsprechend der Normlage wird die Schleifleiste im Bereich $-35 \mathrm{~cm}$ bis $+35 \mathrm{~cm}$ gleichmäßig abgeschliffen. Bei der Prüfstrecke 1 ist die Schleifkohle einseitig mehr abgeschliffen. Bei der Pruifstrecke 2 ist die Schleifleiste in einem sehr kleinen Bereich von $-20 \mathrm{~cm}$ bis $-5 \mathrm{~cm}$ sehr stark abgeschliffen worden.

\begin{tabular}{|c|c|c|c|c|c|}
\hline$m$ & \multicolumn{4}{|c|}{ Bereichsdatenansicht } & \\
\hline \multicolumn{6}{|c|}{ Dateiname: berlin lda } \\
\hline \multicolumn{6}{|c|}{ Pfad: d-Yborldchquel_winttextdat } \\
\hline \multicolumn{6}{|c|}{ Schleifkohlenbereich : 100 [mun] } \\
\hline Nr: & Pos.1 [Y1] & Pos $2[Y 2]:$ & Y2-Y1 : & & \\
\hline \multirow{14}{*}{$\begin{array}{l}0 \\
1 \\
2 \\
3 \\
4 \\
5 \\
6 \\
7 \\
8 \\
9 \\
10 \\
11 \\
12 \\
13 \\
14\end{array}$} & 3294.64 & 3326.72 & 32.72 & \pm & \\
\hline & 3567.69 & 3599.31 & 31.62 & $\square$ & \\
\hline & 4415.90 & 4447.85 & 31.95 & & \\
\hline & 6916.74 & 6948.52 & 31.78 & & Qrucken \\
\hline & 3366.43 & 3398.68 & 32.25 & & \\
\hline & $\begin{array}{l}8378.23 \\
9570.14\end{array}$ & $\begin{array}{l}8403.20 \\
9594.74\end{array}$ & $\begin{array}{l}24.97 \\
24.60\end{array}$ & & \\
\hline & 8834.93 & 8857.55 & 22.62 & & Speichern unter \\
\hline & 954.88 & 1110.42 & 15.55 & & \\
\hline & 7419.97 & 7435.30 & 15.33 & & \\
\hline & 4743.17 & 4760.30 & 17.13 & & Sneichern \\
\hline & 5718.10 & 5734.90 & 16.80 & & Speichern \\
\hline & $\begin{array}{l}1655.30 \\
4383.23\end{array}$ & $\begin{array}{l}1671.06 \\
4399.53\end{array}$ & $\begin{array}{l}15.76 \\
16.30\end{array}$ & & \\
\hline & 2503.05 & 2519.94 & 16.89 & & DK. \\
\hline & & & & +4 & Abbrechen \\
\hline+1 & & & & T & \\
\hline
\end{tabular}

Bild 4 Fahrleitungs- Zick-Zack kleiner gleich $100 \mathrm{~mm}$

Bei einer anderen Art der Auswertung können die Fahrleitungsbereiche angezeigt und ausgedruckt werden, bei denen der Zick-Zack über eine längere Fahrstrecke kleiner als beispielsweise $100 \mathrm{~mm}$ ist (Bild 4). Hier erfolgt die Stromentnahme über eine längere Fahrstrecke/ Zeit über die selbe Stelle auf der Schleifleiste. Die Schleifkohle wird in diesen Streckenabschnitten elektrisch (thermisch) und mechanisch ïberlastet. Die Folgen wurden bereits beschrieben.

Bei der Suche nach der Ursache von Querrillen in der Schleifkohle wird die Lage der Querrille im Kohlekörper

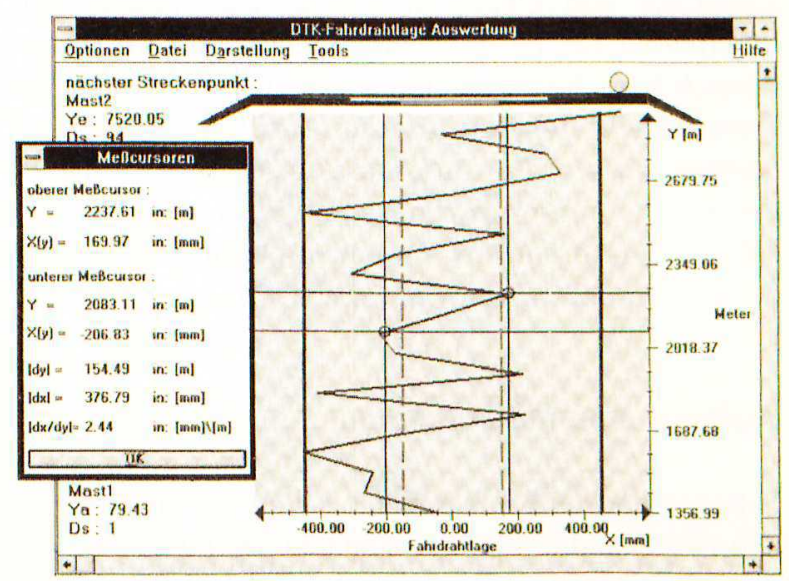

Bild 5 Lage der Fahrleitung im Schleifkohlebereich $-50 \mathrm{~mm}$ bis $+50 \mathrm{~mm}$ 
in das Programm eingegeben (z.B. $-50 \mathrm{~mm}$ bis $50 \mathrm{~mm}$ ). Der Computer ermittelt dann den Fahrleitungsabschnitt, in dem der elektrische Kontakt über diesen Kohleabschnitt längere Zeit erfolgt (Bild 5).

Zur Ermittlung der Lageänderung wird der gewünschte Streckenabschnitt ausgewählt. Der Cursor 1 wird auf den Anfang des Abschnittes der Cursor 2 auf das Ende des Abschnittes gesetzt. Am Bildrand kann dann sofort die Lageänderung als Quotient aus Streckenabschnitt in Fahrtrichtung (dx) zur Seitenlage (dy) abgelesen werden (Bild 6).

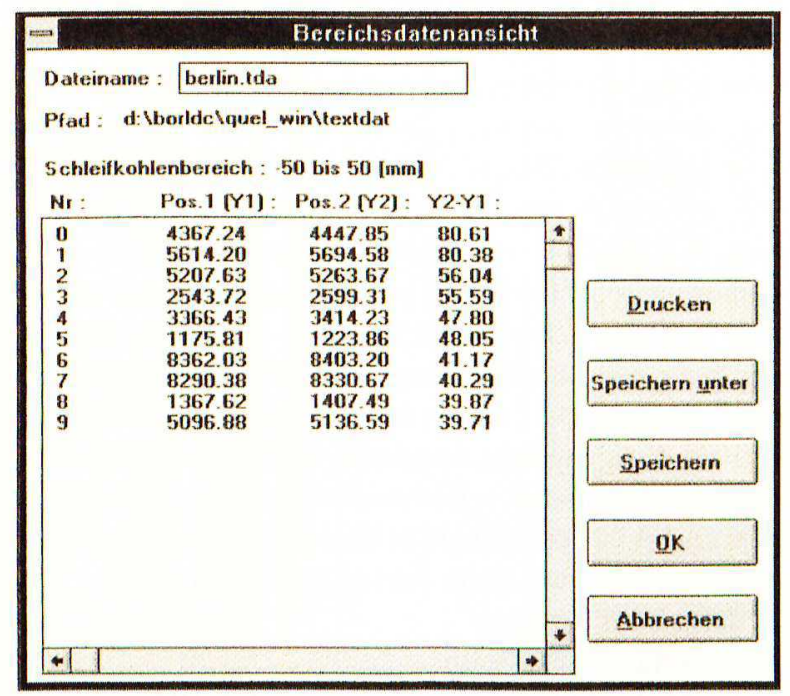

Bild 6 Lageänderung der Fahrleitung

\subsubsection{Höhenlage}

Die Bestimmung der Höhenlage der Fahrleitung erfolgt gleichzeitig mit der Ermittlung der Zick-Zack-Lage. Bei der Datenerfassung können die unterschiedlichsten Meßwerterfassungen eingesetzt werden.

Die Aufzeichnung der Daten erfolgt mit einem PC und der Software „Zick-Zack-Lage“.

\subsubsection{Fahrdynamische Eigenschaften bei der Stromentnahme} Mit dem System „DTK-fahrdynamische Eigenschaften“ werden die Anpreßkräfte der Schleifleiste gegen die Fahrleitung unter normalen Fahrbedingungen bei beliebigen Linienfahrzeugen ermittelt. Das vorhandene Stromabnehmersystem wird dabei nicht beeinflußt. Das System arbeitet nach dem bewährten DTK-Meßprinzip. Es entfallen aufwendige Arbeiten für Aufbau, Isolierung und dergleichen. Die Datenaufnahme erfolgt mit Computer bei gleichzeitiger Videoaufnahme. Es wurde bei der Entwicklung besonderer Wert darauf gelegt, daß das System alle Anforderungen für die Durchfuihrung von Dienstleistungen erfiillt.

Bei der Untersuchung werden im Fahrbetrieb für jede Schleifleiste einzeln und für jede Seite der Schleifleisten die Anpreßkräfte gegen die Fahrleitung bestimmt. Die Kräfte können in Abhängigkeit von der

- Arbeitshöhe des Stromabnehmers,

- Fahrgeschwindigkeit des Fahrzeuges,

- Beschleunigung des Fahrzeuges,

- eingestellten Anpreßkraft der Schleifleisten und

- Masse der Schleifleisten

ermittelt werden.
Somit sind mit diesem System Aussagen zur

- optimalen Anpreßkraft der Schleifleisten gegen die Fahrleitung,

- maximal möglichen Fahrgeschwindigkeit in kritischen Bereichen, (z.B. Bruickendurchfahrten),

- Arbeitsweise des Stromabnehmers in Abhängigkeit von der Fahrtrichtung,

- Stromverteilung uiber die zwei Schleifleisten,

- ungleichmäßigen Abnutzung der ersten und zweiten Schleifleiste

- optimalen Masse der Schleifleisten und somit zu ihrer Laufleistung

- Ursache von „Rattermerkmalen“ in der Fahrleitung/ Stromschiene,

- Elastizität von Fahrleitung und Stromabnehmer möglich.

Die aufgenommenen Daten können dann in Tabellenkalulationsprogrammen oder in Datenbanken beliebig weiter verarbeitet werden.

\section{Zusammenfassung}

Von DTK wurde das System Stromabnehmer / Schleifkontakt / Fahrleitung untersucht. Es wurden Prüfmethoden entwickelt, mit denen im normalen Fahrbetrieb das Zusammenwirken bestimmt werden kann. Ursachen für Störungen werden mit den DTK-Systemen bereits im Entstehen erkannt und können somit vor einem Schaden beseitigt werden.

\section{Verfasser}

Dipl.-Chem. Manfred Deutzer

Geschäftsfuihrer der DTK Deutzer Technische Kohle $\mathrm{GmbH}$

Technologie- und Grïnderzentrum Wildau

Bahnhofstr. 1 (SMB-Gelände Haus 19), 15745 Wildau

Tel. 03375-508519

\section{Fußnoten}

1 Dipl.-Chem. M. Deutzer DER NAHVERKEHR Heft 3/96

2 Dipl.-Ing. P. Pohl EISENBAHNINGENIEUR Heft 6/96 Seite 24

3 Dipl.-Chem M. Deutzer/ Dipl.-Ing. G. Hillbrand DER NAHVERKEHR Heft 6/94 Seite 72-77

4 Dipl.-Chem. M. Deutzer DER NAHVERKEHR Heft 12/ 94 Seite 61-63

5 Dipl.-Chem. M. Deutzer DER NAHVERKEHR Heft 3/93 Seite 50-53

6 Dipl.-Chem. M. Deutzer Gebrauchsmusteranmeldung Rollennummer 29606346.0

7 Demoversion erhalten Sie bei DTK 